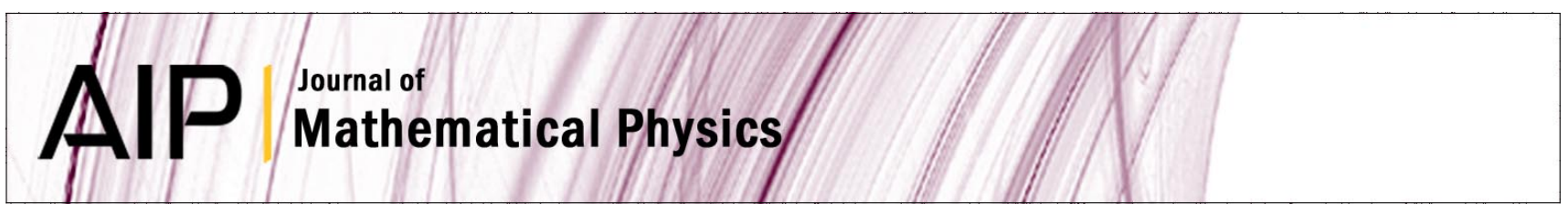

\title{
Thermal field dynamics and bialgebras
}

Tomáš Kopf, A. E. Santana, and F. C. Khanna

Citation: J. Math. Phys. 38, 4971 (1997); doi: 10.1063/1.531928

View online: http://dx.doi.org/10.1063/1.531928

View Table of Contents: http://jmp.aip.org/resource/1/JMAPAQ/v38/i10

Published by the American Institute of Physics.

\section{Related Articles}

Zero and finite temperature Casimir effect of massive vector field between real metals J. Math. Phys. 53, 102302 (2012)

Gröbner bases for finite-temperature quantum computing and their complexity J. Math. Phys. 52, 112203 (2011)

Stochastic quantization of real-time thermal field theory

J. Math. Phys. 51, 102304 (2010)

Finite temperature Casimir effect for a massless fractional Klein-Gordon field with fractional Neumann conditions J. Math. Phys. 48, 082301 (2007)

Finite size effects in bistable 4 models

J. Math. Phys. 48, 012111 (2007)

\section{Additional information on J. Math. Phys.}

Journal Homepage: http://jmp.aip.org/

Journal Information: http://jmp.aip.org/about/about_the_journal

Top downloads: http://jmp.aip.org/features/most_downloaded

Information for Authors: http://jmp.aip.org/authors

\section{ADVERTISEMENT}

The most comprehensive support for physics in any mathematical software package World-leading tools for performing calculations in theoretical physics

www.maplesoft.com/physics
Your work in Maple matches how you would write the problems and solutions by hand

- State-of-the-art environment for algebraic computations in physics

- The only system with the ability to handle a wide range of physics computations as well as pencil-and-paper style input and textbook-quality display of results

- Access to Maple's full mathematical power, programming language, visualization routines, and document creation tools 


\section{Thermal field dynamics and bialgebras}

Tomáš Kopf

Theoretical Physics Institute, Avadh Bhatia Physics Laboratory, University of Alberta, Edmonton, Alberta T6G 2J1, Canada

A. E. Santana

Theoretical Physics Institute, Avadh Bhatia Physics Laboratory, University of Alberta, Edmonton, Alberta T6G 2J1, Canada, and Instituto de Fisica, Universidade

Federal da Bahia, Campus de Ondina, 40210-340, Salvador, Bahia, Brazil

F. C. Khanna

Theoretical Physics Institute, Avadh Bhatia Physics Laboratory, University of Alberta,

Edmonton, Alberta T6G 2J1, Canada, and TRIUMF, 4004 Wesbrook Mall,

Vancouver, British Columbia V6T 2A3, Canada

(Received 3 September 1996; accepted for publication 1 July 1997)

In thermal field dynamics, thermal states are obtained from restrictions of vacuum states on a doubled field algebra. It is shown that the suitably doubled Fock representations of the Heisenberg algebra do not need to be introduced by hand but can be canonically handed down from deformations of the extended Heisenberg bialgebra. No artificial redefinitions of fields are necessary to obtain the thermal representations and the case of arbitrary dimension is considered from the beginning. Our results support a possibly fundamental role of bialgebra structures in defining a general framework for thermal field dynamics. (C) 1997 American Institute of Physics. [S0022-2488(97)02010-0]

\section{INTRODUCTION}

The notion of a doubling structure is present in every theory describing thermal phenomena. This doubling, however, is most transparent in the approach known as thermal field dynamics (TFD). Thermal field dynamics ${ }^{1}$ is based on the idea that thermal states of a quantum system, described by the field algebra $\mathbf{A}$ can be given as restrictions of vacuum states of a doubled algebra of observables $\mathbf{A} \otimes \mathbf{A}$. The doubling of $\mathbf{A}$ is usually given by the so-called tilde conjugation rules which can be thought of as a mapping of $\mathbf{A} \cong \mathbf{A} \otimes 1$ into $1 \otimes \mathbf{A}$ :

$$
\begin{aligned}
(a b)^{\sim}=\tilde{a} \tilde{b}, \quad(\lambda a+\mu b)^{\sim} & =\lambda * \widetilde{a}+\mu^{*} \tilde{b}, \quad(\widetilde{a})^{\sim}=a, \\
\left(a^{+}\right)^{\sim} & =\widetilde{a}^{+}, \\
\mid \text {vacuum }\rangle^{\sim} & =\mid \text { vacuum }\rangle,
\end{aligned}
$$

with $a, b \in \mathbf{A}$ and $\lambda, \mu \in \mathrm{C}$ (the complex numbers).

Such a dual conjugation was proposed as a consequence of the physical analysis of the vacuum-condensation phenomenon, associated with the presence of the unitarily inequivalent vacua of systems with infinite degrees of freedom. This inequivalence is described properly by a Bogoliubov transformation of basic operators of $\mathbf{A}$.

The first attempt to put TFD on an axiomatic basis appeared in Ojima's work ${ }^{2}$ based on the modular (Tomita-Takesaki) conjugation of $C^{*}$-algebras. Such a modular conjugation realizes an omnipresent dual conjugation by switching the algebra $A$ and its commutant $\mathbf{A}^{\prime}$ in the thermal (equilibrium) representation. 
However the explicit splitting of $\mathbf{A} \otimes \mathbf{A}$ into $\mathbf{A} \otimes 1$ and $1 \otimes \mathbf{A}$ is not necessary. For all intended purposes a doubling from $\mathbf{A}$ to $\mathbf{A} \otimes \mathbf{A}$ without a specification of two copies of $\mathbf{A}$ in $\mathbf{A} \otimes \mathbf{A}$ is sufficient and one can therefore in all of the following drop the tilde conjugation rules, retaining only the idea of a doubling.

Note that already at this point the present treatment departs from the different and differently motivated approach to thermal field dynamics based on the modular (Tomita-Takesaki) conjugation as given by I.Ojima. ${ }^{2}$

Given a vacuum state $\omega_{J}$ on $\mathbf{A}$ one can introduce a doubling $\delta_{\chi}: \mathbf{A} \rightarrow \mathbf{A} \otimes \mathbf{A}$ such that the desired thermal state $\omega_{\chi}$ is given by

$$
\omega_{\chi}=\left(\omega_{J} \otimes \omega_{J}\right) \circ \delta_{\chi} .
$$

That this is indeed the case is shown in Section II which also reviews basic facts on the Heisenberg algebra and its exponentiation, the Weyl algebra, mostly omitting proofs. This scheme of producing thermal states has in this setting no deeper justification except that it works.

However, there are two more general points of view.

First, the doubling of the algebra of observables characteristic for thermal field dynamics is just an example of taking tensor products of representations of the field algebra $\mathbf{A}$. A general and systematic way of taking tensor products of representations is given, if $\mathbf{A}$ is equipped with a bialgebra structure, ${ }^{3}$ so it would be nice to have one on our algebra.

Second, a new possibility to study the thermal problem in an algebraic setting has emerged with the works of Celeghini, Vitiello and their co-workers by exploring quantum deformations of Weyl-Heisenberg (WH) in connection with coherent and squeezed states, and quantum dissipation. The fact that one can produce squeezed states and thermal states by deformations of the extended Weyl algebra has been shown using one-dimensional examples ${ }^{4-6}$ and the use of the coalgebra structure as the doubling in thermal field theory was discussed in Refs. 7-9. In particular, a coherent state representation has been exploited and applications to lattice quantum mechanics have been suggested. ${ }^{5,6}$ These works on q-groups and thermal phenomena have set the question of how fundamental the doubling structure for the thermal systems is. A preliminary answer to this question can be given through the notion of Lie symmetries. ${ }^{10}$ In that approach a part of the structure of the theory is deduced from a bialgebra (eventually produced from a symmetry Lie algebra ${ }^{10,11}$ ) with the rest given by the requirement of a Fock structure. The Fock structure itself can, however, also be cast into a bialgebra form by using the extended Heisenberg algebra (see Section III) thus allowing an axiomatic setting of the theory starting only from a bialgebra.

The present treatment is a further development of these results. It deals with the case of a higher dimensional phase space in a rather covariant way. The results are then, in particular, in the right form to be used, e.g., for the description of quantum fields on a curved spacetime background where there is no preferred Hamiltonian to split the phase space into separate modes.

Both points of view presented above ask for a bialgebra. Unfortunately, the Heisenberg algebra A cannot be turned into one as shown in Section III. However, it turns out that by going over to a slightly different algebra, the extended Heisenberg algebra $\mathbf{U}$, one can do away with this problem, and there is a mapping from the extended Heisenberg algebra $\mathbf{U}$ onto the Heisenberg algebra $\mathbf{A}$ that allows one to transport interesting structures, particularly the comultiplication $\Delta$ responsible for tensor products of representations, into the context of physical observables.

This is true even if one q-deforms the extended Heisenberg bialgebra: The deformed extended Heisenberg algebra $\mathbf{U}_{\chi}$ maps down onto the undeformed Heisenberg algebra $\mathbf{A}$ thus giving nothing new for the algebra but providing us with new possible doublings $\Delta_{\chi}$. It is these new doublings $\Delta_{\chi}$ coming from deformations of the extended Heisenberg algebra that allow us to express an arbitrary quasifree state in a way similar to thermal field dynamics. This is shown in Section III together with a discussion of the relevant deformations.

An example showing the relationship between the deformation parameter $\chi$ and the inverse 
temperature of the corresponding thermal state is given in Section IV. Section V contains conclusions and some general remarks.

\section{THE HEISENBERG ALGEBRA AND ITS WEYL FORM}

In order to set the notation it will be shown now that thermal states on a Heisenberg algebra A can be obtained from a Fock representation (i.e., an irreducible representation obtainable from a vacuum $\omega_{J}$ by the Gelfand-Naimark-Segal-construction (GNS-construction, see, e.g., that in O. Bratteli and D.W. Robinson ${ }^{12}$ ) by a suitable ad hoc doubling. The Heisenberg algebra is generated from a symplectic vector space $\Gamma$ of arbitrary dimension with the symplectic form $\sigma(\boldsymbol{\bullet}, \mathbf{0})$ by the usual commutation relations:

$$
\phi\left(z_{1}\right) \phi\left(z_{2}\right)-\phi\left(z_{2}\right) \phi\left(z_{1}\right)=i \hbar \sigma\left(z_{1}, z_{2}\right), \quad z_{1}, z_{2} \in \Gamma
$$

or

$$
W\left(z_{1}\right) W\left(z_{2}\right)=e^{\left[(i / 2) \sigma\left(z_{1}, z_{2}\right)\right]} W\left(z_{1}+z_{2}\right), \quad z_{1}, z_{2} \in \Gamma .
$$

Here $\phi(z)$ are the field operators and $W(z)$ their exponentiated Weyl form:

$$
W(z)=e^{i \phi(z)} .
$$

A vacuum state $\omega_{J}$ on the field algebra $\mathbf{A}$ is given by a complex structure $J$ on $\Gamma$. On the Weyl generators one has:

$$
\omega_{J}(W(z))=e^{-(1 / 4) z^{\circ} \circ \circ \sigma \circ z},
$$

with contractions between the relevant vectors and tensors indicated by $\circ$. The vacuum state is also fully determined by its two-point function:

$$
\omega_{J}\left(\phi\left(z_{1}\right) \phi\left(z_{2}\right)\right)=\frac{1}{2} z_{1}^{\circ}{ }^{\circ} \sigma^{\circ} z_{2} .
$$

The thermal states to be considered are quasifree and thus correspond to free (i.e., quadratic) field Hamiltonians. For each quasifree state there is by the modular theory ${ }^{13}$ (Kubo-MartinSchwinger theory (KMS theory, see O. Bratteli and D.W. Robinson ${ }^{12,14}$ )) a Hamiltonian with respect to which the state is thermal and therefore one has to show that one can produce any quasifree state of interest by our doubled Fock representations. Any quasifree state corresponding to a positive definite Hamiltonian can be written in the form (compare with O. Bratteli and D.W. Robinson, ${ }^{14}$ p.50):

$$
\omega_{\chi}(W(z))=e^{-(1 / 4) z^{\circ} \operatorname{coth}(\Omega / 2)^{\circ} J \circ \sigma^{\circ} z},
$$

where $\Omega$ is a positive definite operator arising from the diagonalization of the corresponding Hamiltonian $H::^{15}$

$$
H=\Omega \circ J \circ \sigma .
$$

It is known from thermal field dynamics that $\Omega$ can be related to a Bogoljubov operator $\chi$ on $\Gamma$ anticommuting with $J$ by the following relation, giving an alternative parametrization of quasifree states:

$$
\begin{gathered}
\cosh (2 \chi)=\operatorname{coth} \frac{\Omega}{2}, \\
\omega_{\chi}(W(z))=e^{-(1 / 4) z^{\circ} \cosh (2 \chi)^{\circ} J^{\circ} \sigma^{\circ} z}
\end{gathered}
$$


with $\chi$ satisfying

$$
\begin{gathered}
J \circ \chi=-\chi^{\circ} J, \\
\sigma^{\circ} \chi=-\chi^{\circ} \sigma .
\end{gathered}
$$

The promised doubling $\delta_{\chi}$ is now given by the following action on the Weyl generators:

$$
\delta_{\chi}(W(z))=W(\cosh (\chi) z) \otimes W(\sinh (\chi) z) .
$$

One can check now by direct calculation that $\delta_{\chi}$ is an algebra homomorphism and that it produces from the doubled vacuum state $\omega_{J} \otimes \omega_{J}$ the right quasifree state $\omega_{\chi}$ :

$$
\begin{aligned}
\omega_{\chi} & =\left(\omega_{J} \otimes \omega_{J}\right) \circ \delta_{\chi}(W(z)) \\
& =\left(\omega_{J} \otimes \omega_{J}\right)(W(\cosh \chi z) \otimes W(\sinh \chi z)) \\
& =\omega_{J}(W(\cosh \chi z)) \omega_{J}(W(\sinh \chi z))
\end{aligned}
$$

using (2)

$$
=e^{-(1 / 4) z^{\circ} \cosh \chi^{\circ} J^{\circ} \sigma^{\circ} \cosh \chi^{\circ} z} e^{-(1 / 4) z^{\circ} \sinh \chi^{\circ} J^{\circ} \sigma^{\circ} \sinh \chi^{\circ} z}
$$

by $(7),(8)$

$$
=e^{-(1 / 4) z^{\circ}\left(\cosh ^{2} \chi+\sinh ^{2} \chi\right)^{\circ} J^{\circ} \sigma^{\circ} z}
$$

by the identity $\cosh ^{2} \chi+\sinh ^{2} \chi=\cosh 2 \chi$

$$
=e^{-(1 / 4) z^{\circ} \cosh 2 \chi^{\circ} J \circ \sigma^{\circ} z} .
$$

But this is just the quasifree state (6) that is required.

\section{THE EXTENDED HEISENBERG ALGEBRA AND ITS DEFORMATIONS}

One would like to use a bialgebra structure on a field algebra, in particular the Heisenberg algebra $\mathbf{A}$, and by the GNS-construction a vacuum state $\omega_{J}$ giving a Fock representation, to produce a new representation in which the vacuum doubled by the comultiplication $\Delta$ will be a thermal (and thus reducible) state.

There is, however, a problem with this straightforward idea: There is no bialgebra structure on the Heisenberg algebra A. This can be easily seen from the fact that the Heisenberg commutation relations for the field $\phi(z)$ require a commutator to be proportional to the unit of the algebra,

$$
\phi\left(z_{1}\right) \phi\left(z_{2}\right)-\phi\left(z_{2}\right) \phi\left(z_{1}\right)=i \hbar \sigma\left(z_{1}, z_{2}\right) 1 \text {. }
$$

Now, a counit $\varepsilon$ of the bialgebra structure has to vanish on commutators and has to be equal to 1 on the unit of the algebra, which is not possible unless the proportionality constant in the commutation relations (Planck's constant) is zero.

To improve that, the unit 1 of the algebra can be replaced by an abstract central element $H$. Now it is no longer necessary for the counit $\varepsilon$ to be equal to 1 on this central element, the commutation relations can be considered as a Lie algebra and there exists even a Hopf algebra structure on this extended Heisenberg algebra $\mathbf{U}$ which is actually now a universal enveloping algebra of a Lie algebra. ${ }^{16}$ To recover a meaning in the field algebra one can map the extension $\mathbf{U}$ 
onto the plain Heisenberg algebra $\mathbf{A}$. The map $\Delta^{()}: \mathbf{A} \rightarrow \mathbf{A} \otimes \mathbf{A}$ induced from the comultiplication $\Delta: \mathbf{U} \rightarrow \mathbf{U} \otimes \mathbf{U}$ is no longer preserving the algebra unit but it is a morphism of algebras and thus allows a tensor product of representations.

What is gained by considering the extended Heisenberg algebra $\mathbf{U}$ is the possibility of having an underlying bialgebra structure giving a canonical doubling on the algebra of observables $\mathbf{A}$.

Moreover, the extended Heisenberg algebra $\mathbf{U}$ can be deformed without changing the scheme, thus producing new interesting doublings on the Heisenberg algebra. The useful deformations can be found for the one-dimensional case in S. Majid ${ }^{16}$ and G. Vitiello, ${ }^{17}$ written in terms of annihilation and creation operators. Additional information on how to obtain these algebras by contractions from semisimple ones can be found in Ref. 18.

In our case the class of possible deformations will be parametrized by a Bogoljubov operator $\chi$ on the classical phase space $\Gamma$ assuming that a vacuum is given by the choice of a complex structure $J$ on $\Gamma$. The Bogoljubov operator is characterized by anticommuting with the complex structure $J$ as well as with the symplectic form $\sigma$ on $\Gamma: 5,6$

$$
J \circ \chi=-\chi \circ J, \quad \sigma \circ \chi=-\chi \circ \sigma .
$$

Our deformations will break the manifest symplectic group symmetry of the extended Heisenberg algebra $\mathbf{U}$ since $\chi$ is not an invariant under these symmetries. They will be written in terms of a set of R-independent eigenvectors $\left\{z_{i}, J z_{i}\right\}$.

The deformed commutation relations are:

$$
\begin{gathered}
{\left[\phi\left(z_{i}\right), \pi\left(z_{j}\right)\right]=-i \delta_{i j}[2 H]_{\chi_{i}},} \\
{\left[\phi\left(z_{i}\right), H\right]=0,} \\
{\left[\pi\left(z_{i}\right), H\right]=0 \text { where } \pi\left(z_{i}\right):=\phi\left(J z_{i}\right)} \\
\text { and }[x]_{\chi_{i}}:=\frac{\sinh \chi_{i} x}{\sinh \chi_{i}} .
\end{gathered}
$$

The deformed comultiplication $\Delta_{\chi}$ is:

$$
\begin{gathered}
\Delta_{\chi} \phi\left(z_{i}\right)=\phi\left(z_{i}\right) \otimes e^{\chi_{i} H}+e^{-\chi_{i} H} \otimes \phi\left(z_{i}\right), \\
\Delta_{\chi} \pi\left(z_{i}\right)=\pi\left(z_{i}\right) \otimes e^{\chi_{i} H}+e^{-\chi_{i} H} \otimes \pi\left(z_{i}\right), \\
\Delta_{\chi} H=H \otimes 1+1 \otimes H .
\end{gathered}
$$

It can be checked by direct calculation that the comultiplication $\Delta$ preserves the commutation relations and that it is coassociative:

$$
\begin{gathered}
{\left[\Delta_{\chi} \phi\left(z_{i}\right), \Delta_{\chi} \pi\left(z_{i}\right)\right]=-i \delta_{i j}\left[2 \Delta_{\chi} H\right]_{\chi_{i}},} \\
\left(\Delta_{\chi} \otimes \mathbb{1}\right) \circ \Delta_{\chi}=\left(1 \otimes \Delta_{\chi}\right) \circ \Delta_{\chi} .
\end{gathered}
$$

The deformed commutation relations (10)-(12) give rise to an algebra isomorphic to the undeformed one $(\chi=0)$. An isomorphism can be easily established, e.g., by rescaling the generators $\phi\left(z_{i}\right)$ by $2 H /\left(2 H_{\chi_{i}}\right)$. In this sense only the coalgebra structure is nontrivially deformed as can be checked by observing that the cocommutative comultiplication $\Delta_{\chi=0}$ becomes noncocommutative. This point was emphasized by Celeghini, Giachetti, Sorace and Tarlini in Ref. 18. If the Heisenberg algebra is understood as constructed from the classical phase space, i.e., to 
consist not only of the algebra alone but also of the inclusion map of generators $z_{i}$ from the classical phase space, then even the deformation (10)-(12) is nontrivial. A rescaling, e.g., of $\phi\left(z_{i}\right)$ is no longer possible without loosing the normalization of $z_{i}$ with respect to the metric $J \circ \sigma$ or changing the inclusion map.

In the Weyl form the deformed commutation relations and the comultiplication can be written as:

$$
\begin{gathered}
U\left(z_{i}\right) V\left(z_{j}\right)=e^{-i[2 H]} \chi_{i} V\left(z_{j}\right) U\left(z_{i}\right), \\
\Delta_{\chi} U\left(z_{i}\right)=U\left(e^{\chi} z_{i}\right) \otimes U\left(e^{-\chi} z_{i}\right), \\
\Delta_{\chi} V\left(z_{i}\right)=V\left(e^{\chi} z_{i}\right) \otimes V\left(e^{-\chi} z_{i}\right),
\end{gathered}
$$

with:

$$
\begin{gathered}
U\left(z_{i}\right):=e^{i \phi\left(z_{i}\right)}=W\left(z_{i}\right), \\
V\left(z_{i}\right):=e^{i \pi\left(z_{i}\right)}=W\left(J z_{i}\right) .
\end{gathered}
$$

We turn now to the canonical mappings $p, p_{n}$ of the deformed extended Heisenberg algebra $\mathbf{U}_{\chi}$ and its coproducts $\Delta_{\chi}{ }^{n-1} \mathbf{U}_{\chi}$ onto the Heisenberg algebra $\mathbf{A}$ and its tensor products $\mathbf{A}^{\otimes n}$. It will be required that $p, p_{n}$ are algebra homomorphisms and that $H$ as well as $\Delta_{\chi}{ }^{n-1} H$ are mapped by $p, p_{n}$ into the units $1,1^{\otimes n}$. In the following the generators $\phi\left(z_{i}\right), \pi\left(z_{i}\right)$ will be identified with their images $p\left(\phi\left(z_{i}\right)\right), p\left(\pi\left(z_{i}\right)\right)$.

The map $p$ is fully specified and it is thus tempting to set $p_{n}=p^{\otimes n}$, but then $\Delta_{\chi}{ }^{n-1} H$ would be mapped into $n \cdot 1$ instead of 1 . To fix the normalization one has to set:

$$
\begin{aligned}
& p_{n}\left(\Delta_{\chi}{ }^{n-1} \phi\left(z_{i}\right)\right)=\frac{1}{\sqrt{n}} p^{\otimes n}\left(\Delta_{\chi}{ }^{n-1} \phi\left(z_{i}\right)\right), \\
& p_{n}\left(\Delta_{\chi}{ }^{n-1} \pi\left(z_{i}\right)\right)=\frac{1}{\sqrt{n}} p^{\otimes n}\left(\Delta_{\chi}{ }^{n-1} \pi\left(z_{i}\right)\right) .
\end{aligned}
$$

Now $p_{n}$ is also fully specified. The important thing now is that the map $\Delta_{\chi}{ }^{n-1}: \mathbf{U}_{\chi} \rightarrow \mathbf{U}_{\chi}{ }^{\otimes n}$ factors through the maps $p, p_{n}$ as can be checked on the generators. The result is a map

$$
\Delta_{\chi}^{(n-1)}: \mathbf{A} \rightarrow \mathbf{A}^{\otimes n}
$$

which fills in the commutative diagram

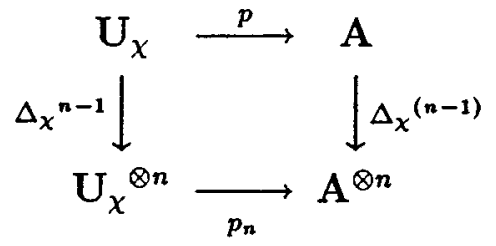

The map $\Delta_{\chi}{ }^{(n-1)}$ is an algebraic homomorphism and thus allows one to take tensor products of representations. Note, however, that due to the necessary normalization $\Delta_{\chi}{ }^{(n-1)}$ is not a comultiplication.

In the case $n=2, \Delta_{\chi}{ }^{(1)}$ is our canonical doubling. If we use now this canonical doubling for doubling a vacuum state $\omega_{J}$ and the corresponding Fock representation, one obtains the state $\omega_{\chi}$ : 


$$
\begin{aligned}
& \omega_{\chi}\left(U\left(z_{i}\right)\right) \equiv \omega_{\chi}\left(W\left(z_{i}\right)\right) \\
& =\left(\omega_{J} \otimes \omega_{J}\right) \circ \Delta_{\chi}{ }^{(1)}\left(U\left(z_{i}\right)\right) \\
& =\left(\omega_{J} \otimes \omega_{J}\right)\left(U\left(e^{\chi} \frac{z_{i}}{\sqrt{2}}\right) \otimes U\left(e^{\chi} \frac{z_{i}}{\sqrt{2}}\right)\right) \\
& =e^{\left.-(1 / 4)(1 / 2) z_{i}{ }^{\circ} e^{\chi_{\circ} J \circ} \sigma^{\circ} e^{\chi_{\circ}} z_{i}\right)} e^{-(1 / 4)(1 / 2) z_{i} e^{\left.-\chi_{\circ J} \circ \sigma^{\circ} e^{-\chi_{\circ}} z_{i}\right)}} \\
& =e^{-(1 / 4)\left(z_{i}^{\circ}\left(e^{2 \chi}+e^{-2 \chi}\right) / 2 \circ J \circ \sigma^{\circ} z_{i}\right)} \\
& =e^{-(1 / 4)\left(z_{i}{ }^{\circ} \cosh 2 \chi^{\circ} J \circ \sigma^{\circ} z_{i}\right)}
\end{aligned}
$$

and similarly:

$$
\omega_{\chi}\left(V\left(z_{i}\right)\right) \equiv \omega_{\chi}\left(W\left(J z_{i}\right)\right)=e^{-(1 / 4)\left(J z_{i} \circ \cosh 2 \chi^{\circ} J \circ \sigma^{\circ} J z_{i}\right)} .
$$

By extension from the generators one gets:

$$
\omega_{\chi}\left(W\left(z_{i}\right)\right)=e^{-(1 / 4)\left(z_{i}^{\circ} \cosh 2 \chi^{\circ} J \circ \sigma^{\circ} z_{i}\right)} .
$$

But this is just the quasifree state (6).

\section{AN EXAMPLE: THE HARMONIC OSCILLATOR}

In the special case of a one-dimensional harmonic oscillator some particular simplifications occur. In its two-dimensional phase space $\Gamma \cong \mathbb{R}^{2}$ there exists a basis in which the symplectic form $\sigma$, the complex structure $J$ and the given Bogoljubov operator $\chi$ take the form:

$$
\begin{aligned}
\sigma_{a b} & =\left(\begin{array}{cc}
0 & 1 \\
-1 & 0
\end{array}\right), \\
J^{a}{ }_{b} & =\left(\begin{array}{cc}
0 & 1 \\
-1 & 0
\end{array}\right), \\
\chi^{a}{ }_{b} & =\left(\begin{array}{cc}
\chi & 0 \\
0 & -\chi
\end{array}\right) .
\end{aligned}
$$

This basis is unique and can be given also by geometrical considerations unless $\chi=0$. Using $J$ as the imaginary unit one can now identify the phase space $\Gamma$ with the complex numbers:

$$
\Gamma \cong \mathrm{C} \text {. }
$$

The vacuum state $\omega_{J}$ and the state $\omega_{\chi}$ obtained in (28) are then given by

$$
\begin{gathered}
\omega_{J}(W(z))=e^{-(1 / 4)\|z\|^{2}}, \\
\omega_{\chi}(W(z))=e^{-(1 / 4) \cosh 2 \chi\|z\|^{2}} .
\end{gathered}
$$

The Hamiltonians compatible with the complex structure $J$ [i.e., those which have a diagonalization (4) giving the fixed $J$ ] are determined by the matrix

$$
H_{a b}=E \beta\left(\begin{array}{cc}
1 & 0 \\
0 & 1
\end{array}\right)
$$


The corresponding thermal states are

$$
\omega_{\beta}(W(z))=e^{-(1 / 4) \operatorname{coth}(\beta E / 2)\|z\|^{2}} .
$$

Fixing $E$ as the energy of the harmonic oscillator and comparing (34) and (36) the states $\omega_{\chi}$ are identified with the thermal states, $\omega_{\beta}$, of the Hamiltonian at inverse temperature $\beta$, obtaining the relation

$$
\operatorname{coth} \frac{\beta E}{2}=\cosh 2 \chi
$$

between the inverse temperature $\beta$ and the deformation parameter $\chi$. So, in the end, the deformation parameter $\chi$ has a nice interpretation as a function of the inverse temperature as pointed out in Ref. 9 and explicitly exhibited here by (37).

\section{CONCLUSION}

It is shown that there is a class of deformations of the extended Heisenberg bialgebra $\mathbf{U}_{\chi}$ that provide canonical doublings by mapping down their comultiplications on the Heisenberg algebra A. These doublings give directly, without any redefinitions, all representations arising from quasifree states by the GNS-construction. In particular, the doublings give all thermal representations for free Hamiltonians. Our construction works clearly for any finite dimensional system and on the level of calculus also for infinite dimensional systems. Functional analytic discussions for the infinite dimensional case are omitted. Note, however, that at no point is unitary equivalence of representations used and that all Bogoljubov transformations are given by symplectomorphisms on the classical phase space. Thus no problem is expected in extending our considerations to the infinite dimensional case.

It would be useful to know if there are deformations other than the ones used here of the extended Heisenberg algebra $\mathbf{U}$. If not, then the construction becomes entirely canonical, since the class of deformations appears to be the only choice one could make in the construction. If there are other deformations, then it would be interesting to see the interpretation of the induced doublings on the Heisenberg algebra $\mathbf{A}$ arising from them.

In any case the present results show that the bialgebra structure is a logical way to approach thermal field theory by providing both the correct results and a mathematically satisfactory general structure.

\section{ACKNOWLEDGMENTS}

F.C.K. and A.E.S. would like to thank G. Vitiello and M. Tuite for useful discussions and T.K. would like to thank T. Brzeziński and P. Krtouš for having explained to him several steps used here and D.N. Page for useful discussions and support. The research is supported in part by Natural Sciences and Engineering Research Council of Canada and by CNPq (a Brazilian Government Agency for Research).

\footnotetext{
${ }^{1}$ Y. Takahashi and H. Umezawa, Collect. Phenom. 2, 55 (1995); H. Umezawa, H. Matsumoto, and M. Tachiki, Thermo Field Dynamics and Condensed States (North Holland, Amsterdam, 1982); H. Umezawa, Advanced Field Theory: Micro, Macro and Thermal Physics (AIP, New York, 1993), and references therein.

${ }^{2}$ I. Ojima, Ann. Phys. 137, 1 (1981).

${ }^{3}$ J. Madore, An Introduction to Noncommutative Geometry and its Physical Applications (London Mathematical Society, Cambridge, 1995).

${ }^{4}$ A. Iorio and G. Vitiello, Mod. Phys. Lett. B 8, 269 (1994).

${ }^{5}$ E. Celeghini, S. de Martino, S. de Siena, G. Vitiello, and M. Rasetti, Mod. Phys. Lett. B 7, 1321 (1993).

${ }^{6}$ E. Celeghini, S. de Martino, S. de Siena, M. Rasetti, and G. Vitiello, Ann. Phys. 241, 50 (1995).

${ }^{7}$ E. Celeghini, M. Rasetti, and G. Vitiello, Ann. Phys. 215, 156 (1992).

${ }^{8}$ E. Celeghini and M. Rasetti, Int. J. Phys. 10, 1625 (1996).
} 
${ }^{9}$ S. de Martino, S. de Siena, and G. Vitiello, Int. J. Phys. 10, 1615 (1996).

${ }^{10}$ A.E. Santana and F.C. Khanna, Phys. Lett. A 203, 68 (1995); A.E. Santana, F.C. Khanna, H. Chu, and Y.C. Chang, Ann. Phys. (N.Y.) 249, 481 (1996); A.E. Santana, T. Kopf, and F.C. Khanna, in Proceedings of the Lake Louise Winter Institute (World Scientific, Singapore, 1996).

${ }^{11}$ H. S. Song, S. X. Ding, and I. An, J. Phys. A 26, 5197 (1993).

${ }^{12}$ O. Bratteli and D.W. Robinson, Operator Algebras and Quantum Statistical Mechanics (Springer, New York, 1979), Vol. I.

${ }^{13}$ M. Takesaki, Tomita's Theory of Modular Hilbert Algebras and its Applications, Lecture Notes in Mathematics, Vol. 128 (Springer, New York, 1970).

${ }^{14}$ O. Bratteli and D.W. Robinson, Operator Algebras and Quantum Statistical Mechanics (Springer, New York, 1981), Vol. II.

${ }^{15} \mathrm{P}$. Krtouš (private communication).

${ }^{16}$ S. Majid, Foundations of Quantum Group Theory (Cambridge University Press, Cambridge, 1995).

${ }^{17}$ G. Vitiello, "The algebraic structure of Thermo Field Dynamics and the $q$-deformation of the Weyl-Heisenberg algebra, 1995," in Proceedings of the Fourth Workshop on Thermal Theories and Their Applications, edited by Y.X. Gui, F.C. Khanna, and Z.B. Su (World Scientific, Singapore, 1996), p.13.

${ }^{18}$ E. Celeghini, R. Giachetti, E. Sorace, and M. Tarlini, J. Math. Phys. 32, 1155 (1991). 